\title{
Radiotherapy combined with hormonal therapy in prostate cancer: the state of the art
}

This article was published in the following Dove Press journal:

Cancer Management and Research

9 October 2010

Number of times this article has been viewed

\section{Piotr Milecki',2 \\ Piotr Martenka' \\ Andrzej Antczak ${ }^{3}$ \\ Zbigniew Kwias ${ }^{3}$}

'Department of Radiotherapy, Greater Poland Cancer Center, Poznan, Poland; ${ }^{2}$ Department of Electroradiology, Medical University, Poznan, Poland;

${ }^{3}$ Chair of Urology, Medical University, Poznan, Poland
Corespondance: Piotr Milecki Department of Radiotherapy, Greater Poland Cancer Center, Garbary I5, 6I-866 Poznań, Poland

$\mathrm{Tel}+48618850877$

Fax +486I8850876

Email piotr.milecki@wco.pl

\begin{abstract}
Androgen-deprivation therapy (ADT) is used routinely in combination with definitive external beam radiation therapy (EBRT) in patients with high-risk clinically localized or locally advanced disease. The combined treatment (ADT-EBRT) also seems to play a significant role in improving treatment results in the intermediate-risk group of prostate cancer patients. On the other hand, there is a growing body of evidence that treatment with ADT can be associated with serious and lifelong adverse events including osteoporosis, cardiovascular disease, diabetes, and many others. Almost all ADT adverse events are time dependant and tend to increase in severity with prolongation of hormonal manipulation. Therefore, it is crucial to clearly state the optimal schedule for ADT in combination with EBRT, that maintaining the positive effect on treatment efficacy would keep the adverse events risk at reasonable level. To achieve this goal, treatment schedule may have to be highly individualized on the basis of the patient-specific potential vulnerability to adverse events. In this study, the concise and evidence-based review of current literature concerning the general rationales for combining radiotherapy and hormonal therapy, its mechanism, treatment results, and toxicity profile is presented.
\end{abstract}

Keywords: prostate cancer, radiotherapy, androgen deprivation, combined treatment

\section{Overview}

Over the past 2 decades, prostate cancer (PC) patients have become the largest cancer population among all cancer patients in the United States and European Union countries. PC is the second and the third leading cause of cancer deaths in males in the United States and European Union countries, respectively. ${ }^{1}$ The most common form of treatment for advanced PC is androgen-deprivation therapy (ADT), which can take the form of either surgical castration (orchiectomy) or medical castration (with luteinizing hormone-releasing hormone [LHRH] agonist). ${ }^{2}$ In addition, more and more patients with unfavorable intermediate- and high-risk adenocarcinoma of the prostate are treated with combination of ADT and primary external beam radiation therapy (EBRT), which seems to be the most appropriate treatment. ${ }^{3-5}$ As shown in a Cancer of the Prostate Strategic Urologic Research Endeavor (CaPSURE) report, the use of neoadjuvant hormones with radiotherapy (RT) increased from $9.8 \%$ to $74.6 \%$ during 1989-1992 and 1999-2001, and from $15.3 \%$ to $89.5 \%$ during the same periods in high-risk patients. ${ }^{6} \mathrm{~A}$ report by Shahinian et al indicated that use of ADT increased steadily throughout the 1990s among men of all ages with PC who had all stages and grades of tumor. ${ }^{7}$ These numbers highlight the need for good understanding of such treatment approach. On the other side, as the role of ADT in the treatment algorithm of PC continues to evolve, there has been increasing attention toward identifying and 
preventing ADT-associated adverse events in recent literature. Common side effects associated with ADT include skeletal complications, metabolic and cardiovascular complications, sexual dysfunction, hot flashes, periodontal disease, cognition, and mood disorders. ${ }^{8,9}$ Some of the abovementioned adverse events can be associated with serious and lifelong disability, morbidity, and possibly mortality. Almost all ADT adverse events are time dependant and tend to increase in severity with prolongation of hormonal manipulation. Therefore, it is crucial to clearly state the optimal schedule for ADT in combination with EBRT, that maintaining the positive effect on treatment efficacy would keep the adverse events risk at a reasonable level. To achieve this goal, treatment schedule may have to be highly individualized on the basis of the patientspecific potential vulnerability to adverse events. In this paper, the concise and evidence-based review of current literature concerning the general rationales for combining RT and hormonal therapy (HT), its mechanism, treatment results, and toxicity profile is presented.

\section{Rationales for combined treatment ADT-EBRT}

In an early work, Huggins and Hodges stated that male hormones promote the growth of both prostate gland and cancer cells. ${ }^{10}$ However, recently it has been recognized that the relationship between serum levels of testosterone and PC progression is not that straightforward. Very low (castrate) concentrations of serum testosterone are enough to fulfill its activating role on the PC cell through the so-called aberrant androgen-signaling pathways. ${ }^{11,12}$ Moreover, there is an evidence for a significant increase in local synthesis of testosterone within prostate stimulated by castrate levels of serum hormone. ${ }^{13}$ Even so, the incomplete eradication of testosterone with surgical or pharmacological castration has proved to improve the results of RT, and multiple studies looked into the possible mechanism and character of that interaction.

The idea that ADT added to RT may improve the results of combined treatment is based on an earlier experience in the application of neoadjuvant HT with surgery. ${ }^{14}$ Neoadjuvant surgical trials have shown response rates (reduction of prostate volume, down-staging, and reduction of positive margin) approaching 90\% when hormonal treatment prior to surgery was administered. However, this modality only reduces the number of positive postoperative margins after radical prostatectomy (RP), but without any influence on overall patient survival. ${ }^{15}$ The mechanism whereby HT and RT interact has been studied in vivo in animal models.
In the original article by Zietman et al, the Shionogi in vivo tumor system has provided the basis for our understanding of the mechanism behind interplay between ADT and RT. The tumors, designed to mimic PC, were implanted into severe combined immune-deficient mice. ${ }^{16}$ Then radiation combined with hormonal manipulation (orchiectomy) was performed at varying time sequences relative to each other. The results showed that ADT improved the tumor response to radiation, which was reflected by reduction in the RT dose needed to control $50 \%$ of tumors (TCD50). Moreover, it was observed that when orchiectomy was performed prior to RT, much lower doses of radiation were required to achieve the given level of tumor control than if performed after or during RT. The authors conclude that increased overall cell kill seems to be a mechanism responsible for the combined effects of ADT and RT. Therefore, the decrease in the number of clonogenic cancer cells due to androgen-ablation therapy should enhance the effects of RT in tumors at the same dose range. ${ }^{17}$ In another in vivo study, RT and androgen-ablation sequencing were evaluated in the R33270G Dunning rat prostate tumor model. ${ }^{18}$ It was found that the median posttreatment tumor doubling time was significantly longer in the group that received RT after neoadjuvant androgen ablation compared to all the other treatment groups, including the RT with concurrent or adjuvant androgen-deprived groups. The possible mechanism of ADT-EBRT interaction in that study was the diminished growth velocity of the surviving PC cells after neoadjuvant ADT.

Other more clinical observations shed some light on ADT-EBRT interactions. Generally, androgen deprivation combined with RT is thought to influence the results of treatment due to local and/or systemic actions. However, whether the increased efficacy of ADT-EBRT is the result of an improved local treatment (radiosensitizing effect) or systemic eradication of micrometastases or the combination of both remains an unanswered question. Obviously, ADT leads to shrinkage of the entire prostate gland volume. It has very practical implications for RT. First, the field dimensions used in RT can be smaller, thereby allowing administration of a higher total dose without increased side effects to healthy tissue. ${ }^{19}$ Data from numerous studies indicate that neoadjuvant HT results in substantial tumor volume reduction, ranging from $30 \%$ to $40 \% .{ }^{20}$ Another postulated mechanism refers to possible enhanced oxygenation (and related increased loco-regional RT effectiveness) of the hypoxic PC cells by improving blood flow with decreasing interstitial pressure when the total amount of cancer cells in the tumor is diminished by ADT. ${ }^{21}$ It is also plausible that apoptosis 
induced by HT could affect cancer cells in which apoptosis was not activated by RT. ${ }^{22}$ The apoptotic mechanism could also be relevant for the systemic interaction of ADT-EBRT by prevention of the subsequent distant micro metastases. The other systemic interaction scenario includes an increase in tumoricidal immune system response, which has been postulated to be a low-androgen-dependent state. ${ }^{23}$

\section{Efficacy of EBRT-ADT combinations Low-risk PC patients}

There is probably no role for ADT in men with low risk for biochemical recurrence PC (T1-T2a, Gleason score [GS] 6 or below, prostate-specific antigen [PSA] below 10). Nevertheless, large number of patients with localized PC are receiving ADT as primary or neoadjuvant treatment, yet the clinical evidence do not support the use of such treatment. As reported by Lu-Yao et al, the ADT as a primary treatment for low-risk PC does not improve survival. ${ }^{24}$ Generally, for low-risk PC patients, no published mature data on combined EBRT-ADT treatment exist from prospective randomized clinical trials. However, the first results of Radiation Therapy Oncology Group (RTOG) 94-08 randomized trial, which completed accrual in 2001 and has been designed to ascertain whether men with stage T1b-T2 PC and a serum PSA of $20 \mathrm{ng} / \mathrm{mL}$ or less benefit from the addition of ADT given for 4 months before and concomitantly with EBRT, has been presented as late-breaking news on recent ASTRO 2009, in November 2009. ${ }^{25}$ This landmark study, with 1,979 participants, is the largest PC study to date among studies evaluating ADT-EBRT and is still ongoing. Originally, the study was designed to evaluate the treatment of men with low-risk PC only, but the definition of low risk evolved as the study got underway. About one-third of the patients were at low risk $(n=685)$, which was defined as a GS of 6 or less with a PSA level of $10 \mathrm{ng} / \mathrm{mL}$ or less and a tumor stage of T2a or less. About one half of the patients were at intermediate risk $(\mathrm{n}=1068)$, which was defined as a GS of 7 , a GS of 6 or less and a PSA of 10-20 ng/mL, or a GS of 6 or less and stage $\mathrm{T} 2 \mathrm{~b}$ disease. The remaining patients were at high risk $(\mathrm{n}=226)$, with GS of $8-10$. Study participants were randomized to short-term ADT (2 months before and 2 months during radiation) plus radiation therapy, or radiation therapy alone. At 8 years, the overall and diseasespecific survival rates in low-risk patients treated with hormones and radiation were comparable to those treated with radiation alone. Specifically, the overall survival (OS) rate at 8 years for patients treated with hormones and radiation was $76 \%$, compared with $73 \%$ for those treated with radiation alone (hazard ratio [HR], 1.07; 95\% confidence interval [CI]: 0.83-1.39). The disease-specific survival rate at 8 years for patients treated with hormones and radiation was $98 \%$, compared with $99 \%$ for those treated with radiation alone (HR, 1.07; 95\% CI: 0.83-1.39).

Practically, no data or conclusions for low-risk PC patients can be pooled out of previous EBRT-ADT trials because most of them consisted of patients with T2C or higher stage tumors. In a study conducted by Bolla et al T1-T2 patients were also included, though only if they were also of GS 7-10, which also refers to a higher risk group than that deemed low risk according to National Comprehensive Cancer Network classification. ${ }^{26}$

Besides the RTOG trial, the only available evidence for the use of combined treatment can be sought in retrospective studies. D'Amico and associates reported results of a large retrospective study $(\mathrm{N}=1586)$ of men treated with 3D-CRT plus or minus ADT for low-risk, intermediate-risk, and highrisk $\mathrm{PC} .{ }^{27}$ In this study, the median radiation dose was 70.2 Gy, and ADT was used in 276 men for 2 months before radiation therapy, during treatment, and for 2 months after treatment was completed. With a median follow-up of 51 months, the 5-year PSA relapse-free survival for men with low-risk PC was $92 \%$ with the addition of ADT vs $84 \%$ without ADT $(P=0.09)$. The issue of RT-HT in low-risk PC patients was also indirectly addressed by a retrospective analysis conducted by Ciezki et al. ${ }^{28}$ The study included 1,668 patients with low- and intermediate-risk PC treated at The Cleveland Clinic Foundation with EBRT, RP, or prostate brachytherapy with or without androgen deprivation during 1996-2001. The 5-year biochemical recurrence-free survival (BRFS) rate was $90 \%$ vs $93 \%$ for EBRT alone or with ADT in low-risk patients.

\section{Intermediate-risk PC patients}

None of the completed prospective randomized clinical trials in PC have directly addressed the usage of EBRT-ADT combination in the group of intermediate-risk patients. However, results of three large randomized trials in which intermediate-risk patients constituted a significant percentage have been published or announced during last few years.

Most recently, data from RTOG study 94-08, which sought to determine whether 8-week neoadjuvant ADT improves RT outcome for patients with clinical stage II PC with a low to intermediate relapse risk, were presented on ASTRO 51 in Chicago. ${ }^{29}$ About $54 \%$ of the patients accrued for that trial were at intermediate risk $(n=1068)$, which 
was defined as a GS of 7, a GS of 6 or less and a PSA of 10-20 ng/mL, or a GS of 6 or less and stage T2b disease. In the study, total androgen suppression was achieved with flutamide $250 \mathrm{mg}$ twice daily and either goserelin $3.6 \mathrm{mg}$ once a month or leuprolide $7.5 \mathrm{mg}$ once a month. The HT apparently benefited men with intermediate-risk disease. At 8 years, the overall and disease-specific survival rates were favorable in intermediate-risk patients treated with hormones and radiation, compared with those treated with radiation therapy alone. Specifically, the OS rate at 8 years for patients treated with hormones and radiation was $72 \%$, compared with $66 \%$ for those treated with radiation alone (HR, $1.23 ; 95 \%$ CI: 1.02-1.49). The disease-specific survival rate at 8 years for patients treated with hormones and radiation was $98 \%$, compared with $92 \%$ for those treated with radiation alone (HR, 2.44; 95\% CI: 1.47-4.04).

The DFCI 95096 trial by D'Amico et al consisted of a limited course of androgen deprivation combined with radiation $(\mathrm{N}=206)$ for clinically localized PC (GS $>7$ or a serum PSA $>10 \mathrm{ng} / \mathrm{mL}$ or evidence of extraprostatic disease). Patients were randomized to receive radiation therapy to a dose of 70 Gy alone or 70 Gy radiation plus 6 months of ADT. Approximately $60 \%$ of patients were at intermediate risk. At a median follow-up of 4.52 years, men treated with the combination of radiation plus androgen deprivation had a significantly higher OS than men treated with radiation alone (actuarial 5-year survival is $88 \%$ vs $78 \%$ ). ${ }^{30}$ What is special about this trial is that all-cause mortality estimates were stratified by randomized treatment group and further stratified in a postrandomization analysis by the comorbidity score. In the latest update with a median follow-up of 7.6 (range, 0.5-11.0) years, 74 deaths have occurred. A significant increase in the risk of all-cause mortality (44 vs 30 deaths; HR, 1.8; 95\% CI: $1.1-2.9 ; P=0.01)$ was observed in men randomized to EBRT compared with EBRT-ADT. However, the increased risk in all-cause mortality appeared to apply only to men randomized to EBRT with no or minimal comorbidity (31 vs 11 deaths; HR, 4.2; 95\% CI: 2.1-8.5; $P<0.001)$. Among men with moderate or severe comorbidity, those randomized to EBRT alone vs EBRT-ADT did not have an increased risk of all-cause mortality (13 vs 19 deaths; HR, 0.54; 95\% CI: $0.27-1.10 ; P=0.08)$. The authors concluded that the addition of 6 months of ADT to EBRT resulted in increased OS in men with localized but unfavorable-risk PC and that this result may pertain only to men without moderate or severe comorbidity. ${ }^{31}$

The third trial conducted was performed by The TransTasman Radiation Oncology Group - TROG 96.01. There were 802 men accrued with locally advanced PC of which approximately $20 \%$ fell into definition of intermediate-risk PC. ${ }^{32}$ The trial had three arms, patients were randomized to RT alone, 3 months of neoadjuvant hormones with RT, or 6 months of neoadjuvant hormones with RT. The protocol prescription was 66 Gy to the prostate and seminal vesicles, without whole pelvic RT. Five-year PSA disease-free survival was significantly improved in the both 3-month arm $(52 \% ; P=0.002)$ and 6-month arm $(56 \% ; P<0.0001)$ as compared to the control arm (38\%). However, the 6-month arm (94\%) showed significantly improved prostate-cancerspecific survival (PCSS; 0.56 [0.32-0.98]; $P=0.04$ ) compared with no androgen deprivation, while for the 3-month arm, the PCSS (92\%) was not significantly different from the control arm (91\%). Interestingly, also 5-year distant failures were significantly less in the 6-month arm (13\%) but not for the 3-month arm $(22 \%)$ as compared to the control $\operatorname{arm}(19 \%)$.

Moreover, some data and conclusions about the efficacy of EBRT-ADT in this group of patients may be derived indirectly from the retrospective subset analyses of previously described randomized clinical trials because some intermediate-risk PC patients were included in RTOG 85-31, European Organisation for Research and Treatment of Cancer (EORTC) 22863, RTOG 86-10, and RTOG 92-02. In all of these trials, investigational arms with EBRT-ADT (or longterm EBRT-ADT vs short-term EBRT-ADT in the case of RTOG 92-02) showed benefit in efficacy end points such as local control (LC), progression-free survival (PFS), BRFS, and incidence of distant metastases. Moreover, the latest update of RTOG 85-31 at the 10-year follow-up showed benefit in OS for all patients in the EBRT-ADT arm. The subset analysis of RTOG 86-10 at 8 years showed improvement in OS for patients with bulky (T2C-T4) tumors but a GS of 2-6.

As for ongoing trials, the definitive results of trial RTOG 99-10, which already completed accrual for randomized Phase III to evaluate the duration of NCHT ( 8 weeks vs 28 weeks) with EBRT in intermediate-risk PC patients, are still awaited. ${ }^{33}$ Also, the RTOG 08-15 study is underway to evaluate more modern high-dose radiation methods and HT in these intermediate-risk patients. ${ }^{34}$

\section{High-risk PC patients}

During the last decade, the results of several prospective randomized clinical trials have indicated that combined treatment (androgen ablation plus RT) leads to improved treatment results. One of these well-documented clinical trials was 
carried out by Pilepich et al. ${ }^{35}$ In this trial (RTOG 8531), the influence of androgen depletion combined with RT on the results of treatment was evaluated. Patients were randomized to receive RT alone or RT plus adjuvant goserelin (LHRH agonist), which was introduced in the last week of RT and continued until the disease progressed or as long as it was tolerated by the patient. RT fields in the first phase of treatment encompassed pelvic lymph nodes, dosed from 44 to $50 \mathrm{~Gy}$, followed by an additional dose of 20-25 Gy to the prostate. Eligible patients had pelvic lymph node involvement (N1) or T3-T4. In the mid 90s, PSA determination became mandatory for all patients participating in the trial. At the median followup time of 4.5 years (range; $0.2-9.8$ years), $84 \%$ of patients on the combined therapy arm and $71 \%$ of those on the RT-alone arm had no evidence of local recurrence $(P<0.0001)$. The update of RTOG trial 85-31 presented in 1999, with a median follow-up time of 5.6 years for all patients and 6 years for patients who were alive, showed an improvement in cause specific survival in the group of patients receiving additional HT treatment $(P=0.019) .{ }^{36}$ The latest update was presented in 2005: after 10 years, there was a $10 \%$ advantage in OS for the HT arm $(P=0.002) .{ }^{37}$ Patients with GS of $7-10$ showed the greatest improvement in survival rate. In the next study (RTOG 8610) carried out by Pilepich et al, patients received neoadjuvant ( 2 months prior to RT) then androgen ablation (goserelin + flutamide) during RT in the study group, and RT alone in the control group. ${ }^{38}$ The RT technique was similar to that applied in trials RTOG 8307 and 8531 . The results of this trial indicated that patients in the combined therapy group had better LC, with 5- and 8-year failure rates of 25\% and $37 \%$, respectively, compared with $36 \%$ and $49 \%$ in the RT-alone group $(P<0.002)$. The most recent analysis of RTOG 86-10 indicated that in patients with GS 7-10, the regimen has not resulted in a significant improvement in either loco-regional control or survival. However, in patients with GS 2-6 tumors, short-course HT administered before and during RT resulted in a highly significant improvement in OS $(70 \%$ vs $52 \%, P=0.015) .{ }^{39}$ An interesting analysis combining the RTOG 85-31 and RTOG 86-10 trials was performed by Horwitz et al. ${ }^{40}$ According to this study, the statistically significant benefit in bNED control $(P=0.0002)$, DMF $(P=0.05)$, and CSF $(P=0.02)$ in patients receiving long-term HT was limited to centrally reviewed GS 7 and 8-10 tumors.

The results of the next well-documented randomized trial conducted by the EORTC come from Europe. In this study, which was reported by Bolla et al, long-lasting adjuvant HT during follow-up was compared to follow-up without additional $\mathrm{HT}^{41}$ In the first phase of this trial, goserelin acetate (LHRH analogue) and cyproterone acetate ( $150 \mathrm{mg} / \mathrm{d} / 1 \mathrm{mo}$ ) were administered prior to RT concurrently during RT in both arms of the study. Thereafter, only in the investigational arm, androgen depletion therapy (LHRH analogue) was continued for 3 years. Patients in both groups received a 50 Gy dose of radiation to the pelvic lymph nodes and then an additional $20 \mathrm{~Gy}$ dose to the prostate. The results of this study were particularly noteworthy because it compared short neoadjuvant androgen ablation treatment with long-term adjuvant therapy. This trial indicated that LC in the investigational arm (combined treatment) was $97 \%$ compared to $77 \%$ in the control arm (no further treatment after RT) at the 45-month follow-up. The 5-year OS in the combined treatment arm was $79 \%$ vs $62 \%$ in the RT-alone group, respectively.

Another important study, reported by Laverdiere et al compared the following three treatment methods: RT alone, neoadjuvant-combined androgen blockade ( 3 months $)+$ RT, and neoadjuvant-combined androgen blockade (3 months) + RT + adjuvant-combined androgen blockade (10.5 months). ${ }^{42}$ The results of this study showed the advantage of neoadjuvant and adjuvant HT over RT alone. The study found that patients treated with a 64 Gy dose in a combined fashion noted $28 \%$ positive biopsies compared to $65 \%$ treated with RT alone. However, the androgen deprivation given 3 months before and 6 months after the RT was associated with only a 5\% rate of positive biopsies. Data concerning the influence of combined therapy on treatment outcome are also based on observation of 1,554 patients entered in trial RTOG 9202 conducted by Hanks et al. ${ }^{43}$ According to the trial protocol, all the patients received goserelin and eulexin 2 months before and then during RT. After completion of RT, they were randomized without any further therapy or were administered additional goserelin alone for 24 months. The study showed that significant improvement in local progression rate $(6.2 \%$ vs $13 \%)$, disease-free survival (54\% vs $34 \%$ ), freedom from distant metastases (11\% vs 17\%), and biochemical control (46\% vs $21 \%$ ) was achieved in the group of patients who were treated long-term hormonally. It should be emphasized that subset analyses (T3, T4, and T2 with GS $8-10$ ) showed no significant OS difference ( $77 \%$ vs $80 \%$ ) over 5 years.

One of the latest randomized clinical trials is the Early PC (EPC) program, the largest treatment trial of patients with localized or locally advanced PC. The program is helping to define which patients benefit, and which do not, from early or adjuvant anti-androgen therapy. Third analysis results, 
at 7.4-years median follow-up, were recently released. The program comprises three randomized, double-blind, placebo-controlled trials designed for combined analysis. Men $(\mathrm{n}=8,113)$ with localized $(\mathrm{T} 1-2, \mathrm{~N} 0 / \mathrm{Nx})$ or locally advanced (T3-4, any N; or any T, N+) PC (all M0) were recruited. Patients received bicalutamide $150 \mathrm{mg}(\mathrm{n}=4,052)$ or placebo $(n=4,061)$ once daily plus standard care $(R T, R P$, or watchful waiting [WW]). The primary endpoints were OS and objective PFS. Bicalutamide significantly improved OS in patients with locally advanced disease who received RT (HR, $0.65 ; P=0.0276)$; this was driven by a lower risk of death due to PC (16.1\% vs $24.3 \%)$. The ongoing EPC program sheds light on the role of anti-androgen therapy and indicates significant clinical benefit from the addition of bicalutamide $150 \mathrm{mg}$ to standard care for patients with locally advanced disease; in particular, an OS benefit was seen in men who received $\mathrm{RT}^{44}$

During the last decades, there were a few papers published, which showed that long-term adjuvant ADT combined with RT for high-risk PC group is related with significant improvement in OS. On the other hand, because long-term ADT caused several side effects, Bolla et al have conducted a randomized clinical trial (EORTC 22961, the so-called Bolla's second trial) in which investigators compared the effects of a shorter treatment regimen (6 months of ADT) to long-term regimen (36 months of ADT). ${ }^{45}$ In this non-inferiority trial, 1,113 patients were included, but 970 patients underwent randomization. Criteria for participation in this trial included T1c to $\mathrm{T} 2 \mathrm{a}-\mathrm{b}, \mathrm{pN} 1$ or $\mathrm{pN} 2, \mathrm{M} 0$ or with $\mathrm{T} 2 \mathrm{c}$ to $\mathrm{T} 4, \mathrm{cN} 0$ to $\mathrm{cN} 2$, and M0, baseline level of PSA up to 40 times the upper limit of the normal range, and a World Health Organization performance status of $0-2$. The first 6 months of androgen suppression consisted of complete androgen blockade (CAB) with an LHRH analogue, initiated on the first day of irradiation, and an antiandrogen agent ( $750 \mathrm{mg}$ of flutamide per day or $50 \mathrm{mg}$ of bicalutamide per day), initiated 1 week before the start of treatment with the LHRH analogue. The patients assigned to long-term suppression continued to be treated with the same LHRH analogue but without the antiandrogen for another 2.5 years. RT was applied in the first phase to the whole pelvis (50 Gy) and then to the prostate gland up to $70 \mathrm{~Gy}$ in both arms. Investigators indicated that after 6.4 years of followup, a 5 years, overall mortality was higher with short-term ADT than with long-term ADT, as well PC-specific mortality increased by $3.8 \%$ and $1.5 \%$, respectively. So, in conclusion of this trial, authors stated that a long-term ADT combined with RT should be the gold standard for high-risk PC patients.

\section{ADT toxicity \\ Sexual dysfunction}

The typical and first described adverse effects of ADT are impotence and loss of libido. The relationship between androgen ablation and sexual function has been studied in several series. ${ }^{46}$ In one series, Potosky et al compared men selecting WW $(n=416)$ with men selecting ADT $(n=245)$ during the first year following cancer of the prostate $(\mathrm{CaP})$ diagnosis. ${ }^{47}$ Patients completed sexual and quality of life surveys at baseline, 6 months postdiagnosis, and 12 months postdiagnosis. Among men reporting some sexual interest at baseline, $54 \%$ of the ADT group vs $13 \%$ of the WW group reported no interest in sexual activity at approximately 12 months postdiagnosis $(P<0.001)$. Among men who were potent at baseline, $80 \%$ of the ADT group compared to $60 \%$ of the WW reported impotence at 1-year follow-up $(P<0.001)$. Fowler et al compared health-related quality of life (HRQOL) outcomes in androgen-deprived $(n=298)$ and nonandrogen-deprived men $(n=1,095)$ following RP in a survey-based study using Medicare Provider and Analysis and Review files. Overall, 166 men in the ADT group and 886 men in the non-ADT group responded to the survey questions regarding erectile dysfunction. Patient receiving ADT reported higher rates of postprostatectomy impotence ( $72 \%$ vs 55\%), but similar rates of impotence over the month prior to the survey ( $23 \%$ vs $22 \%$ ). Regarding the quality of erections, $3 \%$ (vs 11\%) of androgen-deprived men reported erections insufficient for intercourse, and only $2 \%$ (vs $12 \%$; $P<0.0001)$ reported erections firm enough for intercourse. With regard to libido, 69\% (of the 170 responders) in the ADT group reported no sexual drive over the 30 days prior to the survey compared to $29 \%$ (of the 888 responders) in the non-ADT group $(P<0.0001){ }^{48}$

\section{Quality of life}

Currently, no Level I evidence exists that clearly demonstrates association of ADT with a decreased HRQOL, and no consensus recommendations are published to minimize HRQOLrelated adverse effects. Several series have documented an association between ADT and declining HRQOL. ${ }^{49}$ For example. Dacal et al compared HRQOL between men undergoing short-term ADT ( $<6$ months), long-term ADT ( $>6$ months), and healthy controls. When using the MOS questionnaire, they found that men receiving any duration of ADT demonstrated significantly worsened HRQOL. In particular, ADT recipients demonstrated decreased scores in physical component health summary $(P<0.001)$, physical function domain $(P<0.001)$, and general health category $(P<0.001)$. 
Notably, a time-dependent relationship between decreased HRQOL and duration of ADT was not established. ${ }^{50}$ These findings have been supported by other studies demonstrating the negative impact of ADT on cognition, sexual function, social interaction, role functioning, and an increase in the level of emotional distress. ${ }^{51}$ In addition to effects on overall HRQOL, recent data investigating the association between ADT and psychiatric illness has documented an almost twofold increase in the risk of de novo psychiatric illness following ADT induction.

\section{Vasomotor symptoms "hot flashes"}

The so-called "hot flashes" or, more precisely, vasomotor flushings are a common and well-described treatment toxicity in men undergoing androgen ablation and are one of the most frequently reported adverse consequences of ADT. Spetz et al performed a prospective analysis comparing the incidence of hot flashes in men receiving $\mathrm{CAB}$ to that in men receiving estrogen therapy for treatment of PC. ${ }^{52}$ In this study, in 915 patients with metastatic disease, 458 were treated with polyestradiol phosphate and 457 patients received $\mathrm{CAB}$. Of men receiving $\mathrm{CAB}, 74.3 \%$ reported hot flashes compared to $30.1 \%$ in men receiving estrogen therapy $(P<0.001)$. Further, a significantly greater percentage of men treated with CAB were "greatly distressed" by the hot flashes $(11.3 \%$ vs $2.6 \%, P<0.01)$ and reported at least 4 hot flashes per day $(33.7 \%$ vs $2.7 \%, P<0.001)$. ADT-associated vasomotor flushing remains a common complaint reported by men receiving this therapy and is reported in up to $80 \%$ of men receiving ADT. Interestingly, megestrol acetate has been demonstrated to reduce hot flash symptoms by up to $85 \%$. On the other hand, chills, weight gain, and carpal tunnel-like pain are the reported side effects of megestrol acetate. ${ }^{53}$

\section{Endocrine dysfunction and metabolic syndrome}

Male hypogonadism is recognized as an independent risk factor for the development of endocrine dysfunction. ${ }^{54,55}$ In particular, there is increasing evidence supporting an association between ADT and increased risk of the metabolic syndrome and its associated adverse endocrine and end-organ effects. ${ }^{56}$ Metabolic syndrome is diagnosed when three of five criteria proposed by the Adult Treatment Panel III are met, including fasting plasma glucose $>100 \mathrm{mg} / \mathrm{dL}$, serum triglyceride level $>150 \mathrm{mg} / \mathrm{dL}$, serum high-density lipoprotein (HDL) $<40 \mathrm{mg} / \mathrm{dL}$, waist circumference $>102 \mathrm{~cm}$, and blood pressure $>130 / 85$. In one study, a significantly higher overall prevalence of abdominal obesity $(P=0.007)$, hyperglycemia
$(P=0.007)$, and hypertriglyceridemia $(P=0.06)$ in ADT group was noted - all factors that contribute to the diagnosis of metabolic syndrome. Further, the prevalence of the metabolic syndrome was found to be significantly higher in the men receiving ADT (55\%) compared to both the non-ADT group $(22 \%)$ and eugonadal controls $(20 \%, P=0.03)$. The insulin resistance is a major factor of the metabolic syndrome and has also been associated with ADT. ${ }^{57}$ In the study by Smith et al, 25 men with locally advanced or recurrent $\mathrm{CaP}$ and no evidence of metastasis or diabetes were studied for ADT-related effects on insulin resistance. Patients received a 12-week course of CAB (leuprolide depot and bicalutamide) and baseline, and follow-up comparisons were made between the following parameters: plasma glucose, plasma insulin, hemoglobin A1c, lipid profiles, and percentage of body fat. Mean percent body fat mass increased $4.3 \% \pm 1.3 \%$ $(P=0.002)$ after 3 months, while percent lean body mass decreased $1.4 \% \pm 0.5 \%(P=0.006)$. Further, ADT demonstrated significant effects on all of the lipid indices assessed, with rises in total cholesterol $(9.4 \% \pm 2.4 \%, P<0.001), \mathrm{HDL}$ cholesterol $(9.9 \% \pm 2.9 \%, P=0.01)$, low-density lipoprotein cholesterol $(8.7 \% \pm 4.7 \%, P=0.09)$, and triglycerides $(23 \% \pm 8.0 \%, P=0.04)$. No changes in fasting blood glucose were seen during the study; however, significant rises were seen in plasma insulin levels $(P=0.04)$ and mean serum HbA1c levels $(P<0.001)$. Further, insulin sensitivity significantly decreased by nearly $13 \%(P=0.02)$, and one patient was diagnosed with diabetes mellitus (DM) at the completion of the study. ${ }^{58}$ In another series of 73,196 men with local and regional CaP from the Surveillance, Epidemiology, and End Results (SEER) database, a significant increase in the incidence of DM was noticed in men receiving ADT $(P<0.001)$ when compared to those not receiving ADT. Further, the duration of ADT was identified as a predictor for increased risk of subsequent diabetes, even in patients receiving only short courses of ADT.

\section{Osteoporosis and skeletal fractures}

Osteoporosis in men has gained significant clinical attention over the last decade. T-score criteria for the diagnosis of osteoporosis and osteopenia are still evolving; however, it is estimated that using fractures as a clear endpoint for the disease, males have a 13\%-25\% lifetime risk of developing osteoporosis. Hypogonadism is well described as one of the major causes of osteoporosis in men along with alcohol abuse, glucocorticoid excess, low-dietary calcium, vitamin $\mathrm{D}$ deficiency, and sedentary lifestyle. The increasing use of ADT in current practice patterns for the treatment of local 
and advanced $\mathrm{CaP}$ has made ADT one of the leading causes of hypogonadism and thus osteoporosis in men. ${ }^{59,60}$ In a contemporary series of 395 men receiving ADT, Malcolm et al identified ADT as an independent risk factor for the development of osteoporosis and nonpathologic fractures. In this series, $23 \%$ of men receiving ADT developed osteoporosis, while $7 \%$ were diagnosed with nonpathologic fractures. Further, duration of ADT was identified to be an independent predictor for the development of osteoporosis $(P<0.001)$ and was on average $49 \%$ longer in patients diagnosed with fractures $(P<0.001)$. Importantly, the development of osteoporosis was positively associated with the development of nonpathologic fractures in this cohort $(P<0.001)$. Another study brought evidence that gonadotropin-releasing hormone $(\mathrm{GnRH})$ agonists increase the risk of fractures in men receiving ADT when compared to controls. ${ }^{61}$ Shahinian et al analyzed men with $\mathrm{CaP}$ from the SEER database to assess osteoporosis and fracture risk in the ADT population. ${ }^{62}$ For men surviving at least 5 years from $\mathrm{CaP}$ diagnosis, the incidence of fractures was $19.4 \%$ for patients treated with GnRH agonists vs $12.6 \%$ for men treated with other modalities $(P<0.001)$. Further, Cox proportional-hazards regression analyses identified a statistically significant relationship between the number of GnRH injections in the first year following diagnosis and the risk for developing fractures, after adjusting for other clinicopathologic variables. Smith et al assessed the risk for fracture development in men with nonmetastatic disease who were treated with ADT. ${ }^{63}$ Nearly 4,000 men with a history of ADT receipt were matched to men receiving no form of castration therapy $(n=7,774)$. Their comparison study demonstrated a significantly higher clinical fracture risk in the $\mathrm{GnRH}$ agonist group (7.88/100 vs $6.51 / 100$ person-years at risk, $P<0.001)$. Further, ADT independently predicted future fracture risk in multivariate analyses, and longer treatment duration conferred a greater risk for subsequent fractures. The increased fracture rates in patients receiving HT are multifactorial, including higher incidence of metastatic bony lesions, fragility from disease and disease-related treatment, and decreased bone mineral density associated with ADT. ${ }^{64}$

\section{Cardiovascular disease}

Several retrospective studies suggested increased risk for cardiovascular disease in patients receiving ADT. In a study by Malcolm et al, 395 men receiving ADT were reviewed for incidence of cerebrovascular accident or myocardial infarction. Logistic regression demonstrated a time-dependent relationship between risk of myocardial infarction (HR, 2.12; $P=0.03$ ) and cerebrovascular accident (odds ratio $=3.22$, $P=0.001)$ and increasing duration of ADT administration. ${ }^{65}$ Further in another study, Keating et al identified a higher incidence of coronary heart disease $(\mathrm{HR}=1.16, P<0.001)$, myocardial infarction $(\mathrm{HR}=1.11, P=0.03)$, and sudden cardiac death $(\mathrm{HR}=1.16, P=0.004)$ in men receiving ADT when compared to a control group. Additionally, the increased risk of coronary heart disease remained significantly increased even in men receiving ADT for as few as $1-4$ months $(\mathrm{HR}=1.29, P<0.001)$. Myocardial infarction and sudden cardiac death also occurred at higher frequencies in the ADT group when stratified by duration of therapy, though this did not demonstrate statistical significance. Tsai et al directly examined the relationship between ADT and cardiac-related death in an analysis of the CaPSURE database. ${ }^{66}$ Of 4,892 patients with organ-confined CaP, 1,015 received either neoadjuvant or adjuvant ADT, with median therapy duration of 4.1 months, in conjunction with local surgical or radiation treatment. Competing regression analyses that controlled for age, ADT administration, and a history of heart disease or diabetes mellitus at baseline were used to compare cardiac-related mortality rates between men receiving ADT or treated without castration. The authors found that in men treated with RP $(\mathrm{n}=3,262)$, age $(\mathrm{HR}=1.07$, $P=0.003)$ and ADT use $(\mathrm{HR}=2.6, P=0.002)$ were significantly associated with an increased risk of cardiac-related death. Moreover, 5-year cumulative incidence estimates of cardiac death were higher in men receiving ADT when stratified by age ( $P=0.02$ for $<65$ years, $P=0.01$ for $>65$ years). On the other hand, results of a recently completed EORTC randomized trial (Protocol 22961) comparing RT plus a total of 6 months of ADT to RT plus a total of 3 years of ADT in patients with locally advanced PC detected no significant difference in the incidence of fatal cardiac events at 5-year follow-up ( $4.0 \%$ vs 3.0\%, respectively). Moreover, the recent systematic review performed by panel of specialist recommended that at present, based on the available evidence, it can only be stated that ADT may be related with cardiovascular disease risk. ${ }^{67}$

\section{Remarks and conclusions}

In the high-risk group of patients, combined treatment (RT-HT) produced therapeutic gain. For intermediate-risk patients, we have the first results of clinical trials RTOG 94-08, suggesting a statistically significant benefit. More precise conclusion can be made after the results of RTOG 99-10 trial. For low-risk PC patients, combined EBRT-ADT has no 
role in contemporary treatment guidelines. On the basis of the data reviewed from the literature, it can be concluded as follows:

1. ADT is easy to administer and requires no special technology. Neoadjuvant, concurrent, and long-term adjuvant androgen deprivation is standard treatment in conjunction with radiation therapy in the group of patients with high risk of failure (T3, PSA $>20 \mathrm{ng} / \mathrm{mL}, \mathrm{GS}>7$ ). Neoadjuvant, concurrent ADT, and short-term adjuvant should be individually decided in intermediate-risk patients.

2. The optimal timing for application of androgen depletion has not yet been precisely determined. According to the current state of knowledge, approximately 2-3 months for neoadjuvant therapy is probably the optimal strategy. The best mode of neoadjuvant HT is represented by chemical castration combined with short antiandrogen treatment in the initial phase. Neoadjuvant HT should always be followed by ADT concurrent with RT. Adjuvant HT is recommended for high-risk patients for at least 2 years, but longer treatment could be beneficial, provided the toxicity of ADT is not of concern because of patient comorbidities. Studies have shown a survival benefit for patients with more advanced disease when longer adjuvant androgen suppression treatment was applied (3 years).

\section{Disclosure}

The authors report no conflicts of interest in this work.

\section{References}

1. Jemal A, Siegel R, Ward E, et al. Cancer statistics, 2009. CA Cancer J Clin. 2009;59:225-249.

2. Sharifi N, Gulley JL, Dahut WL. Androgen deprivation therapy for prostate cancer. JAMA. 2005;294:238-244.

3. Widmark A, Klepp O, Kolberg A, et al. Endocrine treatment, with or without radiotherapy, in locally advanced prostate cancer (SPCG-7/ SFUO-3): and open randomized phase III trial. Lancet. 2009;373: 301-308.

4. Milecki P, Kwias Z. Hormonal therapy combined with radiotherapy in locally advanced prostate cancer. Rep Pract Oncol Radiother. 2002;7:157-163.

5. Shelley MD, Kumar S, Coles B, Wilt T, Staffurth J, Mason MD. Adjuvant hormone therapy for localised and locally advanced prostate carcinoma: a systematic review and meta-analysis of randomised trials. Cancer Treat Rev. 2009;35:540-546.

6. Cooperberg MR, Grossfeld GD, Lubeck DP, Carroll PR. National practice patterns and time trends in androgen ablation for localized prostate cancer. J Natl Cancer Inst. 2003;95:981-989.

7. Shahinian VB, Kuo Y, Freeman JL, Orihuela E, Goodwin JS. Increasing use of gonadotropin-releasing hormone agonists for the treatment of localized prostate carcinoma. Cancer. 2005;103:1615-1624.

8. Shahinian VB, Kuo YF, Freeman JL, Goodwin JS. Risk of the "androgen deprivation syndrome" in men receiving androgen deprivation for prostate cancer. Arch Intern Med. 2006;166:465-471.

9. Guise TA, Oefelein MG, Eastham JA, Cookson MS, Higano CS, Smith MR. Estrogenic side effects of androgen deprivation therapy. Rev Urol. 2007;9:163-180.
10. Huggins C, Hodges C. Studies on prostatic cancer: the effect of castration, of estrogen, and androgen injection on serum phosphatases in metastatic carcinoma of the prostate. Cancer Res. 1941;1: 293-297.

11. Chen Y, Clegg NJ, Scher HI. Anti-androgens and androgen-depleting therapies in prostate cancer: new agents for an established target. Lancet Oncol. 2009;10:981-991.

12. Edwards J, Krishna NS, Grigor KM, Bartlett JM. Androgen receptor gene amplification and protein expression in hormone refractory prostate cancer. Br J Cancer. 2003;89:552-556.

13. Titus MA, Schell MJ, Lih FB, Tomer KB, Mohler JL. Testosterone and dihydrotestosterone tissue levels in recurrent prostate cancer. Clin. Cancer Res. 2005;11:4653-4657.

14. Soloway M, Sharifi R, Wajsman Z, McLeod D, Wood DP Jr, Puras-Baez A. Randomized prospective study comparing radical prostatectomy alone versus radical prostatectomy preceded by androgen blockade in clinical stage B2 (T2bNxM0) prostate cancer. $J$ Urol. 1995; 154:424-428.

15. Aus G, Abrahamsson PA, Ahlren G, et al. Hormonal treatment before radical prostatectomy: a 3-year follow-up. J Urol. 1998;159: 2016-2017.

16. Zietman AL, Prince EA, Nakfoor BM, Park JJ. Androgen deprivation and radiation therapy: sequencing studies using the Shionogi in vivo tumor system. Int J Radiat Oncol Biol Phys. 1997;38: $1067-1070$.

17. Mcconnell JD. Physiologic basis of endocrine therapy for prostatic cancer. Urol Clin North Am. 1991;18:1-7.

18. Kaminski JM, Hanlon AL, Joon DL, Meistrich M, Hachem P, Pollack A. Effect of sequencing of androgen deprivation and radiotherapy on prostate cancer growth. Int J Radiat Oncol Biol Phys. 2003;57:24-28.

19. Zelefsky MJ, Harrison A. Neoadjuvant androgen ablation prior to radiotherapy for prostate cancer: reducing the potential morbidity of therapy. Urology. 1997;49 Suppl 3 A:38-45.

20. Marcenaro M, Sanguineti G, Franzone P, et al. Neoadjuvant androgen deprivation and prostate gland shrinkage during conformal radiotherapy. Int J Radiat Oncol Biol Phys. 2001;51(ASTRO):18-19.

21. Movsas B, Chapman JD, Horwitz EM, et al. Hypoxic regions exist in human prostate carcinoma. Urology. 1999;53:11-18.

22. Isaacs JT, Lundmo PI, Berges R, Martikainen P, Kyprianou N, English HF. Androgen regulation of programmed death of normal and malignant prostatic cells. J Androl. 1992;13:457-464.

23. Roden AC, Moser MT, Tri SD, et al. Augmentation of T cell levels and responses induced by androgen deprivation. J Immunol. 2004;173:6098-6108.

24. Lu-Yao GL, Albertsen PC, Moore DF, et al. Survival following primary androgen deprivation therapy among men with localized prostate cancer. JAMA. 2008;300:173-181.

25. ASTRO 2009, in November 2009 [ASTRO abstract]. RTOG 94-08.

26. Bolla M, Collette L, Blank L, et al. Long-term results with immediate androgen suppression and external irradiation in patients with locally advanced prostate cancer (an EORTC study): a phase III randomised trial. Lancet. 2002;360:103-106.

27. D'Amico AV, Schultz D, Loffredo M, et al. Biochemical outcome following external beam radiation therapy with or without androgen suppression therapy for clinically localized prostate cancer. JAMA. 2000;284:1280-1283.

28. Ciezki JP, Klein EA, Angermeier K, et al. A retrospective comparison of androgen deprivation (AD) vs no $\mathrm{AD}$ among low-risk and intermediate-risk prostate cancer patients treated with brachytherapy, external beam radiotherapy, or radical prostatectomy. Int J Radiat Biol Phys. 2004;60:1347-1350.

29. 2010 Genitourinary Cancers Symposium (GUCS) in San Francisco Abstract 6. 2010 Mar 5.

30. D'Amico AV, Manola J, Loffredo M, et al. 6-month androgen suppression plus radiation therapy vs radiation therapy alone for patients with clinically localized prostate cancer: a randomized controlled trial. JAMA. 2004;292:821-827. 
31. D'Amico AV, Chen MH, Renshaw AA, Loffredo M, Kantoff PW. Androgen suppression and radiation vs radiation alone for prostate cncer: a randomized trial. JAMA. 2008;299:289-295.

32. Denham JW, Steigler A, Lamb DS, et al. Short-term androgen deprivation and radiotherapy for locally advanced prostate cancer: results from the Trans-Tasman Radiation Oncology Group 96.01 randomised controlled trial. Lancet Oncol. 2005;6:841-850.

33. Radiation Theory Oncology Group. RTOG 99-10. A Phase III trial to evaluate the duration of neoadjuvant total androgen suppression (TAS) and radiation therapy (RT) in intermediate-risk prostate cancer. Available from http://www.rtog.org/members/protocols/99-10/99-10.pdf. Accessed 2010 September 27.

34. Radiation Theory Oncology Group. RTOG 08-15. A Phase III prospective randomized trial of dose-escalated radiotherapy with or without short-term androgen deprivation therapy for patients with intermediate risk prostate cancer. Available from http://www.rtog.org/members/ protocols/0815/0815.pdf. Accessed 2010 September 27.

35. Pilepich MV, Caplan R, Byhardt RW, et al. Phase III trial of androgen supression using goserelin in unfavorable-prognosis carcinoma of the prostate treated with definitive radiotherapy: report of Radiation Oncology Group Protocol 85-31. J Clin Oncol. 1997;15: 1013-1021.

36. Lawton CA, Winter K, Murray K, et al. Updated results of the phase III Radiation Therapy Oncology Group (RTOG) trial 85-31 evaluating the potential benefit of androgen suppression following standard radiation therapy for unfavorable prognosis carcinoma of the prostate. Int J Radiat Oncol Biol Phys. 2001;49: 937-946.

37. Miljenko V, Pilepich M, Winter K, et al. Androgen suppression adjuvant to definitive radiotherapy in prostate carcinoma - long-term results of phase III RTOG 85-31. Int J Radiat Oncol Biol Phys. 2005;61: 1285-1290.

38. Pilepich MV, Winter K, Russell AH, et al. Phase III Radiation Therapy Oncology Group (RTOG) trial 86-10 of androgen deprivation before and during radiotherapy in locally advanced carcinoma of the prostate. Proc Am Soc Clin Oncol. 1998; 17:308a.

39. Miljenko V, Pilepich MV, Winter K, et al. Phase III radiation therapy oncology group (RTOG) trial 86-10 of androgen deprivation adjuvant to definitive radiotherapy in locally advanced carcinoma of the prostate. Int J Radiat Oncol Biol Phys. 2001;50: $1243-1252$.

40. Horwitz EM, Winter K, Hanks GE, et al. Subset analysis of RTOG 85-13 and 86-10 indicates an advantage for long-term vs short-term adjuvant hormones for patients with locally advanced nonmetastatic prostate cancer therapy with radiation therapy. Int J Radiat Oncol Biol Phys. 2001;49:947-956.

41. Bolla M, Gonzalez D, Warde P, et al. Improved survival in patients with locally advanced prostate cancer treated with radiotherapy and goserelin. N Engl J Med. 1997;337:295-300.

42. Laverdiere J, Gomez JL, Cusan L, et al. Beneficial effect of combined therapy administered prior and following external beam radiation therapy in localized prostate cancer. Int J Radiat Biol Phys. 1997;37: 247-252.

43. Hanks, Gerald E, Pajak K, et al. Phase III trial of longterm adjuvant adrogen deprivation after neoadjuvant hormonal cytoreduction and radiotherapy in locally advanced carcinoma of the prostate: the Radiation Therapy Oncology Group Protocol 92-02. J Clin Oncol. 2003;21:3972-3978.

44. Tyrell CJ, Payne H, See WA, et al. Bicalutamide ('Casodex') $150 \mathrm{mg}$ as adjuvant to radiotherapy in patients with localized or locally advanced prostate cancer: results from the randomized Early Prostate Cancer Programme. Radiother Oncol. 2005;76: 4-10.

45. Bolla M, de Reijke TM, Tienhoven GV, et al. Duration of androgen suppression in the treatment of prostate cancer. N Engl J Med. 2009; 360:2516-2527.
46. DiBlasio CJ, Malcolm JB, Derweesh IH, et al. Patterns of sexual and erectile dysfunction and response to treatment in patients receiving androgen deprivation therapy for prostate cancer. BJU Int. 2008;102: 39-43.

47. Potosky AL, Reeve BB, Clegg LX, et al. Quality of life following localized prostate cancer treated initially with androgen deprivation therapy or no therapy. J Natl Cancer Inst. 2002;94:430-437.

48. Fowler FJ Jr, McNaughton Collins M, Walker Corkery E, Elliott DB, Barry MJ. The impact of androgen deprivation on quality of life after radical prostatectomy for prostate carcinoma. Cancer. 2002;95: 287-295.

49. Green HJ, Pakenham KI, Headley BC, et al. Quality of life compared during pharmacological treatments and clinical monitoring for nonlocalized prostate cancer: a randomized controlled trial. BJU Int. 2004;93:975-979.

50. Dacal K, Sereika SM, Greenspan SL. Quality of life in prostate cancer patients taking androgen deprivation therapy. J Am Geriatr Soc. 2006; 54:85-90.

51. Green HJ, Pakenham KI, Headley BC, Gardiner RA. Coping and healthrelated quality of life in men with prostate cancer randomly assigned to hormonal medication or close monitoring. Psychooncology. 2002;11: 401-414.

52. Spetz AC, Hammar M, Lindberg B, Spangberg A, Varenhorst E; Scandinavian Prostatic Cancer Group-5 Trial Study. Prospective evaluation of hot flashes during treatment with parenteral estrogen or complete androgen ablation for metastatic carcinoma of the prostate. J Urol. 2001;166:517-520.

53. Holzbeierlein JM. Managing complications of androgen deprivation therapy for prostate cancer. Urol Clin North Am. 2006;33: $181-190$.

54. Derweesh IH, Diblasio CJ, Kincade MC, et al. Risk of new-onset diabetes mellitus and worsening glycaemic variables for established diabetes in men undergoing androgen-deprivation therapy for prostate cancer. BJU Int. 2007;100:1060-1065.

55. Keating NL, O’Malley AJ, Smith MR. Diabetes and cardiovascular disease during androgen deprivation therapy for prostate cancer. J Clin Oncol. 2006;24:4448-4456.

56. Braga-Basaria M, Dobs AS, Muller DC, et al. Metabolic syndrome in men with prostate cancer undergoing long-term androgen-deprivation therapy. J Clin Oncol. 2006;24:3979-3983.

57. Basaria S, Muller DC, Carducci MA, Egan J, Dobs AS. Hyperglycemia and insulin resistance in men with prostate carcinoma who receive androgen-deprivation therapy. Cancer. 2006;106:581-588.

58. Smith MR, Lee H, Nathan DM. Insulin sensitivity during combined androgen blockade for prostate cancer. J Clin Endocrinol Metab. 2006; 91:1305-1308.

59. Looker AC, Orwoll ES, Johnston CC Jr, et al. Prevalence of low femoral bone density in older US adults from NHANES III. J Bone Miner Res. 1997; 12:1761-1768.

60. Smith MR. Androgen deprivation therapy for prostate cancer: new concepts and concerns. Curr Opin Endocrinol Diabetes Obes. 2007; $14: 247-254$

61. Malcolm JB, Derweesh IH, Kincade MC, et al. Osteoporosis and fractures after androgen deprivation initiation for prostate cancer. Can J Urol. 2007;14:3551-3559.

62. Shahinian VB, Kuo YF, Freeman JL, Goodwin JS. Risk of fracture after androgen deprivation for prostate cancer. N Engl J Med. 2005;352: 154-164.

63. Smith MR, Lee WC, Brandman J, Wang Q, Botteman M, Pashos CL. Gonadotropin-releasing hormone agonists and fracture risk: a claimsbased cohort study of men with nonmetastatic prostate cancer. $J$ Clin Oncol. 2005;23:7897-7903.

64. Maillefert JF, Sibilia J, Michel F, Saussine C, Javier RM, Tavernier C. Bone mineral density in men treated with synthetic gonadotropinreleasing hormone agonists for prostatic carcinoma. J Urol. 1999;161: 1219-1222. 
65. Malcolm JB, Diblasio CJ, Womack JH, et al. Association of cerebrovascular accident and myocardial infarction with Androgen Deprivation Therapy. J Urol. 2007;177:200.

66. Tsai HK, D'Amico AV, Sadetsky N, Chen MH, Carroll PR. Androgen deprivation therapy for localized prostate cancer and the risk of cardiovascular mortality. J Natl Cancer Inst. 2007;99:1516-1524.
67. Levine GN, D'Amico AV, Berger P, et al. Androgen-deprivation therapy in prostate cancer and cardiovascular risk: a science advisory from the American Heart association, American Cancer Society, and American Urological Association: endorsed by the American Society for Radiation Oncology. CA Cancer J Clin. 2010;60;194-201.

\section{Publish your work in this journal}

Cancer Management and Research is an international, peer-reviewed open access journal focusing on cancer research and the optimal use of preventative and integrated treatment interventions to achieve improved outcomes, enhanced survival and quality of life for the cancer patient The journal welcomes original research, clinical \& epidemiological studies, reviews \& evaluations, guidelines, expert opinion \& commentary, case reports \& extended reports. The manuscript management system is completely online and includes a very quick and fair peerreview system, which is all easy to use. Visit http://www.dovepress.com/ testimonials.php to read real quotes from published authors.

Submit your manuscript here: http://www.dovepress.com/cancer-management-and-research-journal 